\title{
NUMERICAL ANALYSIS OF THE MFS FOR CERTAIN HARMONIC PROBLEMS
}

\author{
Yiorgos-Sokratis SMYRlis ${ }^{1}$ AND Andreas Karageorghis ${ }^{1}$
}

\begin{abstract}
The Method of Fundamental Solutions (MFS) is a boundary-type meshless method for the solution of certain elliptic boundary value problems. In this work, we investigate the properties of the matrices that arise when the MFS is applied to the Dirichlet problem for Laplace's equation in a disk. In particular, we study the behaviour of the eigenvalues of these matrices and the cases in which they vanish. Based on this, we propose a modified efficient numerical algorithm for the solution of the problem which is applicable even in the cases when the MFS matrix might be singular. We prove the convergence of the method for analytic boundary data and perform a stability analysis of the method with respect to the distance of the singularities from the origin and the number of degrees of freedom. Finally, we test the algorithm numerically.
\end{abstract}

Mathematics Subject Classification. Primary 65N12, 65N38; Secondary 65N15, 65T50, 65 Y99.

Received: July 29, 2003.

\section{INTRODUCTION}

The Method of Fundamental Solutions is a relatively new boundary method for the solution of certain elliptic boundary value problems. The formulation of the method as a numerical technique was first proposed by Mathon and Johnston [11]. The MFS is a meshless method which is very easy to implement and avoids integration on the boundary. The method has been applied to a variety of physical problems in fluid mechanics, acoustics, electromagnetism and elasticity. Comprehensive surveys of the applications of the MFS and related methods can be found in the recent survey articles [3,4,6]. Error estimates and a convergence analysis of the MFS for circular harmonic problems appear in $[8,9]$. Applications of the MFS can also be found in the books by Kolodziej [10], Golberg and Chen [5] and Doicu, Eremin and Wriedt [2].

In [12] we considered the approximation of the solution of Laplace's equation in the disk $\Omega=\left\{\boldsymbol{x} \in \mathbb{R}^{2}:|\boldsymbol{x}|<\right.$ $\varrho$ \} subject to the Dirichlet boundary condition $u=f$. Although this is a very specific problem, it enables us to identify applicability as well as error and stability features of the method which are present in more general cases.

Keywords and phrases. Method of fundamental solutions, boundary meshless methods, error bounds and convergence of the MFS.

1 Department of Mathematics and Statistics, University of Cyprus, PO Box 20537, 1678 Nicosia, Cyprus.

e-mail: smyrlis@ucy.ac.cy; andreask@ucy.ac.cy 
In the MFS, the solution $u$ is approximated by the harmonic function

$$
u_{N}(\boldsymbol{c}, \boldsymbol{Q} ; P)=\sum_{j=1}^{N} c_{j} k\left(P, Q_{j}^{\alpha}\right), \quad P \in \bar{\Omega},
$$

where $\boldsymbol{c}=\left(c_{1}, c_{2}, \ldots, c_{N}\right)^{T}$ and $\boldsymbol{Q}$ is a $2 N$-vector containing the coordinates of the singularities (sources) $Q_{j}^{\alpha}$, $j=1, \ldots, N$, which lie outside $\bar{\Omega}$. The function $k(P, Q)$ is a fundamental solution of Laplace's equation given by

$$
k(P, Q)=-\frac{1}{2 \pi} \log |P-Q|,
$$

where $|P-Q|$ is the distance between $P$ and $Q$. The singularities $Q_{j}^{\alpha}$ are fixed on the boundary $\partial \tilde{\Omega}$ of a disk $\tilde{\Omega}=\left\{\boldsymbol{x} \in \mathbb{R}^{2}:|\boldsymbol{x}|<R\right\}$ concentric to $\Omega$, with $R>\varrho$. A set of collocation points $\left\{P_{i}\right\}_{i=1}^{N}$ is placed on $\partial \Omega$. If $P_{i}=\left(x_{P_{i}}, y_{P_{i}}\right)$, then we take

$$
x_{P_{i}}=\varrho \cos \frac{2(i-1) \pi}{N}, y_{P_{i}}=\varrho \sin \frac{2(i-1) \pi}{N}, \quad i=1, \ldots, N .
$$

If $Q_{j}^{\alpha}=\left(x_{Q_{j}^{\alpha}}, y_{Q_{j}^{\alpha}}\right)$, then

$$
x_{Q_{j}^{\alpha}}=R \cos \frac{2(j-1+\alpha) \pi}{N}, y_{Q_{j}^{\alpha}}=R \sin \frac{2(j-1+\alpha) \pi}{N}, j=1, \ldots, N,
$$

where the positions of the sources differ by an angle $\frac{2 \pi \alpha}{N}$ from the positions of the boundary points and $0 \leq \alpha<1$. In the case $\alpha \neq 0$, we thus have a rotation of the singularities with respect to the boundary points (see $[8,12]$ ). This rotation improves the accuracy of the method when $R-\varrho \ll 1$ (see Figs. 2 and 4 ).

In the version of the MFS with fixed singularities, the coefficients $c$ are determined so that the boundary condition is satisfied at the boundary points $\left\{P_{i}\right\}_{i=1}^{N}$ :

$$
u_{N}\left(\boldsymbol{c}, \boldsymbol{Q} ; P_{i}\right)=f\left(P_{i}\right), \quad i=1, \ldots, N .
$$

This yields a linear system of the form

$$
G^{\alpha} c=f
$$

for the coefficients $\boldsymbol{c}$, where the elements of the matrix $G^{\alpha}$ are given by

$$
G_{i, j}^{\alpha}=-\frac{1}{2 \pi} \log \left|P_{i}-Q_{j}^{\alpha}\right|, \quad i, j=1, \ldots, N .
$$

Clearly $G^{\alpha}$ is a circulant matrix, see [1]. More precisely $G^{\alpha}=\operatorname{circ}\left(g_{1}(\alpha), \ldots, g_{N}(\alpha)\right)$ where

$$
\begin{aligned}
g_{j}(\alpha) & =-\frac{1}{2 \pi} \log \left|P_{1}-Q_{j}^{\alpha}\right| \\
& =-\frac{1}{4 \pi} \log \left(R^{2}-2 R \varrho \cos \left(\frac{2 \pi(j+\alpha-1)}{N}\right)+\varrho^{2}\right),
\end{aligned}
$$

$j=1, \ldots, N$. Thus $G^{\alpha}$ is diagonalizable

$$
G^{\alpha}=U^{*} D U, \quad \text { where } \quad D=\operatorname{diag}\left(\lambda_{1}(\alpha), \ldots, \lambda_{N}(\alpha)\right),
$$


with eigenvalues

$$
\lambda_{j}(\alpha)=\sum_{k=1}^{N} g_{k}(\alpha) \omega^{(k-1)(j-1)}
$$

where $\omega=\mathrm{e}^{2 \pi i / N}$ and $j=1, \ldots, N$. The corresponding normalized eigenvectors are

$$
\boldsymbol{\xi}_{j}=\frac{1}{\sqrt{N}}\left(1, \omega^{j-1}, \omega^{2(j-1)}, \ldots, \omega^{(N-1)(j-1)}\right)^{T}, \quad j=1, \ldots, N,
$$

and they form an orthonormal basis of $\mathbb{C}^{N}$. The matrix $U$ is therefore unitary $\left(U U^{*}=I\right)$ and its conjugate is

$$
U^{*}=\frac{1}{\sqrt{N}}\left(\begin{array}{ccccc}
1 & 1 & 1 & \cdots & 1 \\
1 & \omega & \omega^{2} & \cdots & \omega^{N-1} \\
1 & \omega^{2} & \omega^{4} & \cdots & \omega^{2 N-2} \\
\vdots & \vdots & \vdots & & \vdots \\
1 & \omega^{N-1} & \omega^{2(N-1)} & \cdots & \omega^{(N-1)(N-1)}
\end{array}\right)
$$

The matrix $U$ is known as the Fourier matrix.

Let $\langle\boldsymbol{\zeta}, \boldsymbol{\eta}\rangle=\sum_{k=1}^{N} \zeta_{k} \bar{\eta}_{k}$ be the complex inner product of $\boldsymbol{\zeta}, \boldsymbol{\eta} \in \mathbb{C}^{N}$. Any vector $\boldsymbol{v} \in \mathbb{C}^{N}$, can be expressed as $\boldsymbol{v}=\sum_{k=1}^{N}\left\langle\boldsymbol{v}, \boldsymbol{\xi}_{k}\right\rangle \boldsymbol{\xi}_{k}$ and hence $G^{\alpha} \boldsymbol{v}=\sum_{k=1}^{N} \lambda_{k}(\alpha)\left\langle\boldsymbol{v}, \boldsymbol{\xi}_{k}\right\rangle \boldsymbol{\xi}_{k}$. In particular, when $G^{\alpha}$ is nonsingular

$$
\left(G^{\alpha}\right)^{-1} \boldsymbol{v}=\sum_{k=1}^{N} \frac{1}{\lambda_{k}(\alpha)}\left\langle\boldsymbol{v}, \boldsymbol{\xi}_{k}\right\rangle \boldsymbol{\xi}_{k}
$$

In Katsurada and Okamoto [9], the authors prove the convergence of the unrotated MFS for Laplace's equation in a disk, subject to analytic Dirichlet boundary data. The extension to non-analytic boundary data and to the rotated case is studied by Katsurada [8]. Our approach is different as it is based on the study of the eigenvalues of the matrix $G^{\alpha}$. We first identify the cases when the matrix $G^{\alpha}$ is singular, we then develop an efficient algorithm for the solution of the problem and, using the information we have obtained about the eigenvalues, we prove the convergence of the method. Finally, from the properties of the eigenvalues, we develop a stability analysis for the method with respect to $R$.

This paper is structured as follows: in Section 2 we study the invertibility of the matrix $G^{\alpha}$ and isolate the eigenvalues which might vanish. In Section 3 we propose a modified algorithm which avoids these troublesome eigenvalues. In Section 4 we prove the exponential convergence of the MFS approximate solution $u_{N}$ to the exact solution $u$ for analytic boundary data as $N$ tends to infinity. In Section 5 we provide a stability result, namely we demonstrate that when the radius $R \gg \varrho$, roundoff error is generated. The resulting deterioration in accuracy when $R$ is large, which is contrary to the theoretical predictions, has often been reported in the literature. Finally in Section 6 we test the method numerically on two examples for which the exact solution in known.

\section{Properties of the eigenvalues}

In this section we investigate the properties of the eigenvalues of the matrix $G^{\alpha}$. We divide these eigenvalues in three groups:

(i) the "first" eigenvalue $\lambda_{1}$;

(ii) in the case $N$ is even, the "middle" eigenvalue $\lambda_{\frac{N}{2}+1}$; and

(iii) the remaining eigenvalues. 
In the sequel we shall assume, without loss of generality, that the radius of the disk is $\varrho=1$. Also, because of symmetry, we shall restrict the parameter $\alpha$ in the interval $\left[0, \frac{1}{2}\right]$.

Combining (1.8) with (1.9) we obtain for $j=1, \ldots, N$ that

$$
\lambda_{j}(\alpha)=-\frac{1}{4 \pi} \sum_{k=1}^{N} \mathrm{e}^{\frac{2 \pi i(j-1)(k-1)}{N}} \log \left(R^{2}+1-2 R \cos \left(\frac{2 \pi(k+\alpha-1)}{N}\right)\right) .
$$

Clearly,

$$
\sum_{k=1}^{N} \sin \left(\frac{2 \pi(j-1)(k-1)}{N}\right) \log \left(R^{2}+1-2 R \cos \left(\frac{2 \pi(k-1)}{N}\right)\right)=0,
$$

which leads to the following result:

Lemma 2.1. When $\alpha=0$, the eigenvalues $\lambda_{j}(0), j=1, \ldots, N$, of $G^{\alpha}$ are real and

$$
\lambda_{j}(0)=\lambda_{N-j+2}(0), \quad j=2, \ldots,\left[\frac{N+1}{2}\right] .
$$

When $\alpha \neq 0$, the eigenvalue $\lambda_{1}(\alpha)$ is real, and so is $\lambda_{\frac{N}{2}+1}(\alpha)$, in the case $N$ is even. The remaining eigenvalues are complex with

$$
\lambda_{j}(\alpha)=\bar{\lambda}_{N-j+2}(\alpha), \quad j=2, \ldots,\left[\frac{N+1}{2}\right] .
$$

\subsection{The first eigenvalue}

We are particularly interested in the points where the first eigenvalue vanishes.

Proposition 2.2. The first eigenvalue $\lambda_{1}(\alpha)$ of the matrix $G^{\alpha}$ is given by

$$
\lambda_{1}(\alpha)=-\frac{1}{4 \pi} \log \left(R^{2 N}-2 R^{N} \cos (2 \pi \alpha)+1\right) .
$$

Proof. Since $G^{\alpha}$ is circulant, i.e., $G^{\alpha}=\operatorname{circ}\left(g_{1}(\alpha), g_{2}(\alpha), \ldots, g_{N}(\alpha)\right)$, the eigenvalues of $G^{\alpha}$ are given by (1.9). In particular,

$$
\lambda_{1}(\alpha)=\sum_{k=1}^{N} g_{k}(\alpha)
$$

From (1.8), we have

$$
\lambda_{1}(\alpha)=-\frac{1}{4 \pi} \log \left\{\prod_{k=1}^{N}\left(R^{2}+1-2 R \cos \left(\frac{2 \pi(k+\alpha-1)}{N}\right)\right)\right\} .
$$

From [7] (p. 34)

$$
\prod_{k=0}^{n-1}\left\{x^{2}-2 x y \cos \left(\alpha+\frac{2 k \pi}{n}\right)+y^{2}\right\}=x^{2 n}-2 x^{n} y^{n} \cos n \alpha+y^{2 n},
$$

from which (2.3) follows. 
From (2.3) we obtain the following corollary:

Corollary 2.3. The eigenvalue $\lambda_{1}(\alpha)$ vanishes if and only if $\alpha=\frac{1}{2 \pi} \cos ^{-1}\left(\frac{R^{N}}{2}\right)$.

We observe the following:

\section{Remarks 2.1.}

(i) An immediate consequence of Corollary 2.3 is that if $R^{N}>2$ (or equivalently $R>2^{\frac{1}{N}}$ ), the eigenvalue $\lambda_{1}(\alpha)$ cannot vanish.

(ii) In order for $\lambda_{1}(\alpha)$ to vanish we need $2 \cos 2 \pi \alpha>1$, i.e., $0<\alpha<\frac{1}{6}$ or $\frac{5}{6}<\alpha<1$. Since because of symmetry we are only considering values of $\alpha \in\left[0, \frac{1}{2}\right)$, it follows that the eigenvalue $\lambda_{1}(\alpha)$ could only vanish for values of $\alpha \in\left[0, \frac{1}{6}\right]$.

\subsection{The middle eigenvalue $\lambda_{\frac{N}{2}+1}(\alpha)$ when $N$ is even}

The behaviour of the eigenvalue $\lambda_{\frac{N}{2}+1}(\alpha)$ in the case $N=2 M$ is even, is of particular interest. Its corresponding normalized eigenvector is

$$
\boldsymbol{\xi}_{\frac{N}{2}+1}=\frac{1}{N^{1 / 2}}(1,-1,1,-1, \ldots, 1,-1)^{T},
$$

which isolates from the boundary data $\left(f_{1}, \ldots, f_{N}\right)^{T}$ the term

$$
\left(\frac{1}{N^{1 / 2}} \sum_{k=1}^{N}(-1)^{k-1} f_{k}\right) \boldsymbol{\xi}_{\frac{N}{2}+1}
$$

corresponding to noise.

Proposition 2.4. If $N$ is even, then the eigenvalue $\lambda_{\frac{N}{2}+1}(\alpha)$ is given by

$$
\lambda_{\frac{N}{2}+1}(\alpha)=-\frac{1}{4 \pi} \log \frac{R^{N}-2 R^{N / 2} \cos \alpha \pi+1}{R^{N}-2 R^{N / 2} \cos (\alpha+1) \pi+1} .
$$

Proof. Let $N=2 M$. We know that the eigenvalues of $G^{\alpha}$ are given by (1.9); therefore

$$
\begin{aligned}
\lambda_{\frac{N}{2}+1}(\alpha)= & -\frac{1}{4 \pi} \sum_{k=1}^{N}(-1)^{k-1} \log \left(R^{2}-2 R \cos \frac{2 \pi(k+\alpha-1)}{N}+1\right) \\
= & -\frac{1}{4 \pi} \sum_{n=1}^{M} \log \left(R^{2}-2 R \cos \left(2 \pi \frac{n-1}{M}+\frac{\alpha \pi}{M}\right)+1\right) \\
& +\frac{1}{4 \pi} \sum_{m=1}^{M} \log \left(R^{2}-2 R \cos \left(2 \pi \frac{m-1}{M}+\frac{(\alpha+1) \pi}{M}\right)+1\right) .
\end{aligned}
$$

From (2.5) we get that

$$
\begin{aligned}
\lambda_{\frac{N}{2}+1}(\alpha) & =-\frac{1}{4 \pi} \log \left(R^{2 M}-2 R^{M} \cos \alpha \pi+1\right)+\frac{1}{4 \pi} \log \left(R^{2 M}-2 R^{M} \cos (\alpha+1) \pi+1\right) \\
& =-\frac{1}{4 \pi} \log \frac{R^{N}-2 R^{N / 2} \cos \alpha \pi+1}{R^{N}-2 R^{N / 2} \cos (\alpha+1) \pi+1} .
\end{aligned}
$$


A direct application of this theorem yields the following corollary:

Corollary 2.5. For $N$ even and $R>1$, the eigenvalue $\lambda_{\frac{N}{2}+1}(\alpha)$ vanishes if and only if $\alpha=\frac{1}{2}$. [We assume that $\alpha \in[0,1)$. $]$

2.3. The eigenvalues $\lambda_{j}(\alpha), j \neq 1$, and $j \neq \frac{N}{2}+1$, when $N$ is even

Theorem 2.6. For $\alpha \in\left[0, \frac{1}{2}\right]$ and $j \neq 1$, we have

$$
\lambda_{j}(\alpha)=\frac{N}{4 \pi} \sum_{m=0}^{\infty}\left\{\frac{\mathrm{e}^{-i \frac{2 \pi}{N}(m N+j-1) \alpha}}{(m N+j-1) R^{m N+j-1}}+\frac{\mathrm{e}^{i \frac{2 \pi}{N}(m N+N-j+1) \alpha}}{(m N+N-j+1) R^{m N+N-j+1}}\right\} .
$$

In particular when $N$ is even

$$
\lambda_{\frac{N}{2}+1}(\alpha)=\frac{N}{2 \pi} \sum_{m=0}^{\infty} \frac{\cos (2 m+1) \alpha \pi}{\left(m N+\frac{N}{2}\right) R^{m N+\frac{N}{2}}} .
$$

Proof. Let $F(R, \vartheta)=-\frac{1}{4 \pi} \log \left(R^{2}-2 R \cos \vartheta+1\right)$. Then, from [7] we have

$$
F(R, \vartheta)=-\frac{1}{4 \pi} \log \left(R^{2}-2 R \cos \vartheta+1\right)=-\frac{1}{2 \pi} \log R+\frac{1}{2 \pi} \sum_{n=1}^{\infty} \frac{\cos n \vartheta}{n R^{n}} .
$$

Then for $j=2, \ldots, N$, we have

$$
\begin{aligned}
\lambda_{j}(\alpha)= & \sum_{k=1}^{N} \exp \left(i \frac{2 \pi}{N}(j-1)(k-1)\right) F\left(R, \frac{2 \pi}{N}(k-1+\alpha)\right) \\
= & \frac{1}{2 \pi} \sum_{n=1}^{\infty} \frac{1}{n R^{n}}\left\{\sum_{k=1}^{N} \cos \left(\frac{2 \pi}{N}(j-1)(k-1)\right) \cos \left(\frac{2 \pi}{N} n(k-1+\alpha)\right)\right\} \\
& +i \frac{1}{2 \pi} \sum_{n=1}^{\infty} \frac{1}{n R^{n}}\left\{\sum_{k=1}^{N} \sin \left(\frac{2 \pi}{N}(j-1)(k-1)\right) \cos \left(\frac{2 \pi}{N} n(k-1+\alpha)\right)\right\} .
\end{aligned}
$$

We are going to calculate the above two sums. We first observe that:

$$
\begin{aligned}
& \sum_{k=1}^{N} \cos \left(\frac{2 \pi}{N} k \ell\right) \cos \left(\frac{2 \pi}{N} n(\ell+\alpha)\right)=\frac{1}{2} \cos \left(\frac{2 \pi}{N} n \alpha\right)\left\{C_{n-\ell}^{N}+C_{n+\ell}^{N}\right\}, \\
& \sum_{k=1}^{N} \sin \left(\frac{2 \pi}{N} k \ell\right) \cos \left(\frac{2 \pi}{N} n(\ell+\alpha)\right)=\frac{1}{2} \sin \left(\frac{2 \pi}{N} n \alpha\right)\left\{C_{n-\ell}^{N}-C_{n+\ell}^{N}\right\},
\end{aligned}
$$

where

$$
C_{\kappa}^{N}=\sum_{j=1}^{N} \cos \left(\frac{2 \pi}{N} \kappa j\right)=\left\{\begin{array}{rrr}
N & \text { if } & \kappa \equiv 0 \bmod N \\
0 & \text { if } & \kappa \neq 0 \text { mod } N
\end{array}\right.
$$


Combining (2.10), (2.11) and (2.12), and replacing the $C_{\kappa}^{N}$ according to (2.13), we obtain

$$
\begin{aligned}
\lambda_{j}(\alpha) & =\frac{1}{4 \pi} \sum_{n=1}^{\infty} \frac{\mathrm{e}^{-i \frac{2 \pi}{N} n \alpha}}{n R^{n}} C_{n-j+1}^{N}+\frac{1}{4 \pi} \sum_{n=1}^{\infty} \frac{\mathrm{e}^{i \frac{2 \pi}{N} n \alpha}}{n R^{n}} C_{n+j-1}^{N} \\
& =\frac{N}{4 \pi} \sum_{m=0}^{\infty} \frac{\mathrm{e}^{-i \frac{2 \pi}{N}(m N+j-1) \alpha}}{(m N+j-1) R^{m N+j-1}}+\frac{N}{4 \pi} \sum_{m=0}^{\infty} \frac{\mathrm{e}^{i \frac{2 \pi}{N}(m N+N-j+1) \alpha}}{(m N+N-j+1) R^{m N+N-j+1}} .
\end{aligned}
$$

Formula (2.8) is an immediate consequence of (2.7).

In particular, when $\alpha=0$ we have the following formula for the eigenvalues

Corollary 2.7. For $\alpha=0$ and $j \neq 1$, we have

$$
\lambda_{j}(0)=\frac{N}{4 \pi} \sum_{m=0}^{\infty}\left(\frac{1}{j-1+m N} \cdot \frac{1}{R^{j-1+m N}}+\frac{1}{N-j+1+m N} \cdot \frac{1}{R^{N-j+1+m N}}\right) .
$$

In particular, for $N$ even and $j=\frac{N}{2}+1$, we have

$$
\lambda_{\frac{N}{2}+1}(0)=\frac{N}{2 \pi} \sum_{m=0}^{\infty} \frac{1}{\frac{N}{2}+m N} \cdot \frac{1}{R^{\frac{N}{2}+m N}} .
$$

Finally for $j=\left[\frac{N}{2}\right]+1, \ldots, N$, we have $\lambda_{j}(0)=\lambda_{N-j+2}(0)$.

We also have the following two corollaries:

Corollary 2.8. For all $j=2, \ldots, N$ we have $\lambda_{j}(0)>0$.

In particular, since $\lambda_{1}(0)=0$ if and only if $R=2^{\frac{1}{N}}$ (see Cor. 2.3), we also have the corollary:

Corollary 2.9. The matrix $G^{0}$ is nonsingular if and only if $R \neq 2^{\frac{1}{N}}$.

Next we shall prove that the eigenvalues $\lambda_{j}(\alpha)$, for $\alpha \in\left[0, \frac{1}{2}\right]$ and $j=2, \ldots,\left[\frac{N+1}{2}\right]$, never vanish. In particular, we have the following result:

Lemma 2.10. For $\alpha \in\left[0, \frac{1}{2}\right)$ and $j=2, \ldots,\left[\frac{N+1}{2}\right]$, we have $\boldsymbol{R} e \lambda_{j}(\alpha)>0$.

Proof. Let $r_{j}(\alpha)=\boldsymbol{R} e \lambda_{j}(\alpha)$. The proof of the lemma is a consequence of the following two properties of the $r_{j}$ :

(i) for all $\alpha \in\left[0, \frac{1}{2}\right]$ and $j=2, \ldots,\left[\frac{N+1}{2}\right]$, we have that $\frac{\mathrm{d} r_{j}(\alpha)}{\mathrm{d} \alpha}<0$. Therefore, for $\alpha \in\left[0, \frac{1}{2}\right]$, the $r_{j}(\alpha)$ are decreasing functions in the interval $\left[0, \frac{1}{2}\right]$;

(ii) for all $j=2, \ldots,\left[\frac{N+1}{2}\right]$, we have that $r_{j}\left(\frac{1}{2}\right)>0$. Therefore, $r_{j}(\alpha) \geq r_{j}\left(\frac{1}{2}\right)>0$.

Proof of (i):

Clearly

$$
r_{j}(\alpha)=\frac{N}{4 \pi} \sum_{m=0}^{\infty}\left\{\frac{\cos \left(\frac{2 \pi}{N}(m N+j-1) \alpha\right)}{(m N+j-1) R^{m N+j-1}}+\frac{\cos \left(\frac{2 \pi}{N}(m N+N-j+1) \alpha\right)}{(m N+N-j+1) R^{m N+N-j+1}}\right\} .
$$

Differentiating with respect to $\alpha$ we obtain

$$
r_{j}^{\prime}(\alpha)=-\frac{1}{2} \sum_{m=0}^{\infty}\left\{\frac{\sin \left(\frac{2 \pi}{N}(m N+j-1) \alpha\right)}{R^{m N+j-1}}+\frac{\sin \left(\frac{2 \pi}{N}(m N+N-j+1) \alpha\right)}{R^{m N+N-j+1}}\right\} .
$$


Let $z=\frac{\mathrm{e}^{i \frac{2 \pi}{N} \alpha}}{R} ;$ then

$$
r_{j}^{\prime}(\alpha)=-\frac{1}{2} \boldsymbol{I} m\left\{\sum_{m=0}^{\infty}\left(z^{m N+j-1}+z^{m N+N-j+1}\right)\right\}=-\frac{1}{2} \boldsymbol{I} m\left\{\frac{\left(z^{j-1}+z^{N-j+1}\right) \overline{\left(1-z^{N}\right)}}{\left|1-z^{N}\right|^{2}}\right\} .
$$

It thus suffices to show that $\boldsymbol{I} m\left(\left(z^{j-1}+z^{N-j+1}\right) \overline{\left(1-z^{N}\right)}\right)>0$, for $\alpha \in\left[0, \frac{1}{2}\right]$. After some simple calculations we get

$$
\begin{gathered}
\boldsymbol{I} m\left(\left(z^{j-1}+z^{N-j+1}\right) \overline{\left(1-z^{N}\right)}\right)=\boldsymbol{I} m\left\{\left(\frac{\mathrm{e}^{i \frac{2 \pi}{N}(j-1) \alpha}}{R^{j-1}}+\frac{\mathrm{e}^{i \frac{2 \pi}{N}(N-j+1) \alpha}}{R^{N-j+1}}\right)\left(1-\frac{\mathrm{e}^{-i \frac{2 \pi}{N} N \alpha}}{R^{N}}\right)\right\} \\
\quad=\sin \left(\frac{2 \pi}{N}(j-1) \alpha\right)\left(\frac{1}{R^{j-1}}+\frac{1}{R^{2-j+1}}\right)+\sin \left(\frac{2 \pi}{N}(N-j+1) \alpha\right)\left(\frac{1}{R^{N-j+1}}+\frac{1}{R^{N+j-1}}\right) .
\end{gathered}
$$

Both the arguments of the sines, in the above expression, lie in $(0, \pi)$ and therefore both $\sin \left(\frac{2 \pi}{N}(j-1) \alpha\right)$ and $\sin \left(\frac{2 \pi}{N}(N-j+1) \alpha\right)$ are positive. Thus the right hand side of (2.16) is positive, which concludes the proof of $(i)$. Proof of (ii):

Let $\gamma=1 / R$, then we have

$$
\begin{aligned}
r_{j}\left(\frac{1}{2}\right)= & \frac{N}{4 \pi} \sum_{m=0}^{\infty}\left\{\frac{\cos \frac{\pi}{N}(m N+j-1)}{(m N+j-1) R^{m N+j-1}}+\frac{\cos \frac{\pi}{N}(m N+N-j+1)}{(m N+N-j+1) R^{m N+N-j+1}}\right\} \\
= & \frac{N}{4 \pi} \cos \frac{\pi(j-1)}{N} \sum_{m=0}^{\infty}\left\{\frac{\gamma^{2 m N+j-1}}{(2 m N+j-1)}+\frac{\gamma^{(2 m+2) N-j+1}}{((2 m+2) N-j+1)}\right\} \\
& -\frac{N}{4 \pi} \cos \frac{\pi(j-1)}{N} \sum_{m=0}^{\infty}\left\{\frac{\gamma^{(2 m+1) N+j-1}}{((2 m+1) N+j-1)}+\frac{\gamma^{(2 m+1) N-j+1}}{((2 m+1) N-j+1)}\right\} .
\end{aligned}
$$

Clearly $\cos \frac{\pi(j-1)}{N}>0$; thus it suffices to show that for every $m>0$ we have

$$
\frac{\gamma^{2 m N+j-1}}{2 m N+j-1}+\frac{\gamma^{(2 m+2) N-j+1}}{(2 m+2) N-j+1}>\frac{\gamma^{(2 m+1) N-j+1}}{(2 m+1) N-j+1}+\frac{\gamma^{(2 m+1) N+j-1}}{(2 m+1) N+j-1},
$$

or equivalently

$$
\frac{1}{2 m N+j-1}-\frac{\gamma^{N}}{(2 m+1) N+j-1}>\gamma^{N-2 j+2}\left(\frac{1}{(2 m+1) N-j+1}-\frac{\gamma^{N}}{(2 m+2) N-j+1}\right) .
$$

Since $\gamma^{N-2 j+2}<1$, it is sufficient to show that

$$
\frac{1}{2 m N+j-1}-\frac{\gamma^{N}}{(2 m+1) N+j-1}>\frac{1}{(2 m+1) N-j+1}-\frac{\gamma^{N}}{(2 m+2) N-j+1},
$$

or equivalently

$$
\frac{m N-2 j+2}{(2 m N+j-1)((2 m+1)(N-j+1)}>\gamma^{N} \frac{m N-2 j+2}{((2 m+1) N+j-1)((2 m+2)(N-j+1)},
$$

which is clearly true. This concludes the proof of property (ii) and the theorem. 
This leads to the central result of this section:

Theorem 2.11. The matrix $G^{\alpha}$ is singular if and only if

(i) $\alpha=\frac{1}{2 \pi} \cos ^{-1}\left(\frac{R^{N}}{2}\right)$, in which case the first eigenvalue $\lambda_{1}(\alpha)$ vanishes;
or

(ii) $\alpha=\frac{1}{2}$, when $N$ is even. In this case the eigenvalue $\lambda_{\frac{N}{2}+1}(\alpha)$ vanishes.

\section{Remarks 2.2.}

(i) From Remark 2.1(ii) and Theorem 2.11 it follows that, if $\alpha \in\left(\frac{1}{6}, \frac{1}{2}\right)$, then $\operatorname{det} G^{\alpha} \neq 0$;

(ii) for every $R>1, \alpha \in\left[0, \frac{1}{2}\right)$, for sufficiently large $N$, the matrix $G^{\alpha}$ is nonsingular. In particular $G^{\alpha}$ is nonsingular for $N \geq\left[\frac{\log 2}{\log R}\right]+1$

(iii) more detailed proofs of the results presented in this section may be found in [13].

\section{IMPLEMENTATION}

\subsection{Expression of $u_{N}$ in terms of the eigenvalues and eigenvectors of $G^{\alpha}$}

By denoting the approximation $u_{N}(\boldsymbol{c}, \boldsymbol{Q} ; P)$ in $(1.1)$ by $u_{N}(P)$, where $P=(x, y)$, we have

$$
u_{N}(P)=\langle\boldsymbol{c}, \boldsymbol{l}\rangle=\left\langle\left(G^{\alpha}\right)^{-1} \boldsymbol{f}, \boldsymbol{l}\right\rangle,
$$

where

$$
\boldsymbol{l}=\boldsymbol{l}(P)=-\frac{1}{2 \pi}\left(\log \left|P-Q_{1}\right|, \ldots, \log \left|P-Q_{N}\right|\right)^{T}
$$

Since the $\left\{\boldsymbol{\xi}_{k}\right\}_{k=1, \ldots, N}$ form an orthonormal basis of $\mathbb{C}^{N}$, for nonsingular $G^{\alpha}$ we have

$$
\left(G^{\alpha}\right)^{-1} \boldsymbol{f}=\left(G^{\alpha}\right)^{-1} \sum_{k=1}^{N}\left\langle\boldsymbol{f}, \boldsymbol{\xi}_{k}\right\rangle \boldsymbol{\xi}_{k}=\sum_{k=1}^{N} \frac{1}{\lambda_{k}(\alpha)}\left\langle\boldsymbol{f}, \boldsymbol{\xi}_{k}\right\rangle \boldsymbol{\xi}_{k} .
$$

Thus using (1.10) we obtain

$$
u_{N}(P)=\left\langle\left(G^{\alpha}\right)^{-1} \boldsymbol{f}, \boldsymbol{l}\right\rangle=\sum_{k=1}^{N} \frac{1}{\lambda_{k}(\alpha)}\left\langle\boldsymbol{f}, \boldsymbol{\xi}_{k}\right\rangle \overline{\left\langle\boldsymbol{l}, \boldsymbol{\xi}_{k}\right\rangle} .
$$

Remark 3.1. By using Fast Fourier Transforms (FFT), the quantities $\lambda_{k}(\alpha),\left\langle\boldsymbol{f}, \boldsymbol{\xi}_{k}\right\rangle$ and $\left\langle\boldsymbol{l}(P)\right.$, $\left.\boldsymbol{\xi}_{k}\right\rangle$, for $k=$ $1, \ldots, N$, can be evaluated at a cost of $O(N \log N)$ operations.

\subsection{Description of the proposed algorithm}

We will suggest a modification of the method which avoids the presence of the inverses of possible zero eigenvalues in (3.2). Clearly, since the only possibly vanishing eigenvalues are $\lambda_{1}$ and $\lambda_{\frac{N}{2}+1}$, if the boundary condition vector $\boldsymbol{f}$ does not contain the corresponding eigenvectors $\boldsymbol{\xi}_{1}$ and $\boldsymbol{\xi}_{\frac{N}{2}+1}\left(i . e .\left\langle\boldsymbol{f}, \boldsymbol{\xi}_{1}\right\rangle=\left\langle\boldsymbol{f}, \boldsymbol{\xi}_{\frac{N}{2}+1}\right\rangle=0\right)$, then the troublesome terms do not appear in (3.2), and thus the MFS can be used without modification. To treat the general boundary condition vector $\boldsymbol{f}$, when $N$ is even, we decompose it as follows:

$$
f=f^{0}+f^{1}+f^{2}
$$

where

$$
\boldsymbol{f}^{0}=\boldsymbol{f}-\left\langle\boldsymbol{f}, \boldsymbol{\xi}_{1}\right\rangle \boldsymbol{\xi}_{1}-\left\langle\boldsymbol{f}, \boldsymbol{\xi}_{\frac{N}{2}+1}\right\rangle \boldsymbol{\xi}_{\frac{N}{2}+1}, \quad \boldsymbol{f}^{1}=\left\langle\boldsymbol{f}, \boldsymbol{\xi}_{1}\right\rangle \boldsymbol{\xi}_{1}, \quad \boldsymbol{f}^{2}=\left\langle\boldsymbol{f}, \boldsymbol{\xi}_{\frac{N}{2}+1}\right\rangle \boldsymbol{\xi}_{\frac{N}{2}+1},
$$


and

$$
\boldsymbol{\xi}_{1}=\frac{1}{\sqrt{N}}(1,1, \ldots, 1), \quad \boldsymbol{\xi}_{\frac{N}{2}+1}=\frac{1}{\sqrt{N}}(1,-1,1,-1, \ldots, 1,-1) .
$$

We shall solve the boundary value problems, corresponding to $\boldsymbol{f}^{\ell}$, for $\ell=0,1,2$, separately.

- For $\ell=0$ the boundary value problem is solved with the MFS yielding the approximate solution

$$
u_{N}^{0}(P)=\sum_{\substack{2 \leq k \leq N \\ k \neq \frac{N}{2}+1}} \frac{1}{\lambda_{k}(\alpha)}\left\langle\boldsymbol{f}, \boldsymbol{\xi}_{k}\right\rangle \overline{\left\langle\boldsymbol{l}, \boldsymbol{\xi}_{k}\right\rangle} .
$$

- For $\ell=1$ the vector describing the boundary is a constant multiple of the constant vector $(1,1, \ldots, 1)$. Since the constant functions are harmonic, the most natural choice for a solution in this case is the constant solution

$$
u_{N}^{1}(P)=\frac{1}{\sqrt{N}}\left\langle\boldsymbol{f}, \boldsymbol{\xi}_{1}\right\rangle
$$

- For $\ell=2$ the vector $\boldsymbol{f}^{2}$ is a constant multiple of

$$
(1,-1,1,-1, \ldots, 1,-1)
$$

An appropriate solution in this case, i.e., a harmonic function the boundary values of which at the points $\left\{P_{k}\right\}_{k=1}^{N}$, coincide with $\boldsymbol{f}^{2}$, is a suitable multiple of

$$
\boldsymbol{R} e z^{\frac{N}{2}}=\boldsymbol{R} e(x+i y)^{\frac{N}{2}} .
$$

The appropriate solution is thus

$$
u_{N}^{2}(P)=\frac{1}{\sqrt{N}}\left\langle\boldsymbol{f}, \boldsymbol{\xi}_{\frac{N}{2}+1}\right\rangle \boldsymbol{R} e z^{\frac{N}{2}} .
$$

Summarizing, the approximate solution of the modified MFS is taken to be

$$
u_{N}(P)=u_{N}^{0}(P)+u_{N}^{1}(P)+u_{N}^{2}(P) .
$$

In the case when $N$ is odd the part $u_{N}^{2}$ is not present.

This algorithm can thus be performed in $O(N \log N)$ operations.

For $\alpha \in\left(\frac{1}{6}, \frac{1}{2}\right)$, the matrix $G^{\alpha}$ is nonsingular and therefore the algorithm can be applied without subtracting the two troublesome terms.

\section{CONVERGENCE OF THE MFS FOR ANALYTiC BOUNDARY DATA}

In this section we shall show that the (unmodified) MFS approximation $u_{N}$ converges uniformly exponentially fast in the $\|\cdot\|_{\infty}$-norm to the exact solution $u$ of the Dirichlet problem in the unit disk $\Omega$, provided that the boundary data $f=\left.u\right|_{\partial \Omega}$ are analytic or equivalently $u$ can be extended as a harmonic function in an open region $D$ containing $\bar{\Omega}=\left\{(x, y) \mid x^{2}+y^{2} \leq 1\right\}$. In particular, we assume that for some $\beta>0$

$$
\bar{B}(0,1+\beta)=\left\{(x, y) \mid\left(x^{2}+y^{2}\right)^{1 / 2} \leq 1+\beta\right\} \subset D .
$$


If we express $f$ as a Fourier series $f(\vartheta)=\sum_{k \in \mathbb{Z}} \hat{f}_{k} \mathrm{e}^{i k \vartheta}$, then (4.1) implies that

$$
\|f\|_{1+\beta}=\left(\sum_{k \in \mathbb{Z}}\left|\hat{f}_{k}\right|^{2}(1+\beta)^{2|k|}\right)^{\frac{1}{2}}<+\infty .
$$

In particular, we have that for every $k \in \mathbb{Z}$

$$
\left|\hat{f}_{k}\right| \leq\|f\|_{1+\beta}(1+\beta)^{-|k|}
$$

Let $f_{N}$ be the Discrete Fourier Interpolant of $f$ corresponding to the values of $f$ at $\vartheta_{j}=\frac{2 \pi}{N} j, j=1, \ldots, N$. Namely for $N$ even (the case when $N$ is odd can be dealt with similarly)

$$
f_{N}(\vartheta)=\sum_{k=-\frac{N}{2}+1}^{\frac{N}{2}} \varphi_{k} \mathrm{e}^{i k \vartheta}
$$

where the coefficients $\varphi_{k} \in \mathbb{C}, k=-\frac{N}{2}+1, \ldots, \frac{N}{2}$, are chosen so that $f_{N}$ and $f$ agree at the points $\left\{\vartheta_{j} \mid j=\right.$ $1, \ldots, N\}$. Clearly if $\omega=\mathrm{e}^{\frac{2 \pi i}{N}}$, then

$$
\begin{aligned}
\varphi_{k} & =\frac{1}{N} \sum_{j=1}^{N} \omega^{-k j} f\left(\vartheta_{j}\right)=\frac{1}{N} \sum_{j=1}^{N} \omega^{-k j}\left(\sum_{\ell \in \mathbb{Z}} \hat{f}_{\ell} \mathrm{e}^{i \ell \vartheta_{j}}\right) \\
& =\frac{1}{N} \sum_{j=1}^{N} \omega^{-k j}\left(\sum_{\ell \in \mathbb{Z}} \hat{f}_{\ell} \omega^{\ell j}\right)=\sum_{\ell \in \mathbb{Z}} \hat{f}_{\ell}\left(\frac{1}{N} \sum_{j=1}^{N} \omega^{-k j} \omega^{\ell j}\right) \\
& =\sum_{m \in \mathbb{Z}} \hat{f}_{k+m N} .
\end{aligned}
$$

Thus

$$
\begin{aligned}
\left|\varphi_{k}\right| & \leq \sum_{m \in \mathbb{Z}}\left|\hat{f}_{k+m N}\right| \leq\|f\|_{1+\beta} \sum_{m \in \mathbb{Z}}(1+\beta)^{-|k+m N|} \\
& \leq 2|| f\left\|_{1+\beta}(1+\beta)^{-|k|} \sum_{n=0}^{+\infty}(1+\beta)^{-n N}=2|| f\right\|_{1+\beta}(1+\beta)^{-|k|} \frac{1}{1-(1+\beta)^{-N}} \\
& \leq \frac{2(1+\beta)}{\beta}\|f\|_{1+\beta}(1+\beta)^{-|k|}=M_{1}\|f\|_{1+\beta}(1+\beta)^{-|k|}
\end{aligned}
$$

with $M_{1}$ depending only on $\beta$. This interpolant converges to $f$ exponentially fast, with respect to the supremum norm, as $N$ tends to infinity. In fact

$$
\begin{aligned}
\left|f_{N}(\vartheta)-f(\vartheta)\right| & =\left|\sum_{k \in \mathbb{Z}} \hat{f}_{k} \mathrm{e}^{i k \vartheta}-\sum_{k=-\frac{N}{2}+1}^{\frac{N}{2}} \varphi_{k} \mathrm{e}^{i k \vartheta}\right| \leq \sum_{k=-\frac{N}{2}+1}^{\frac{N}{2}}\left|\hat{f}_{k}-\varphi_{k}\right|+\sum_{|k| \geq \frac{N}{2}}\left|\hat{f}_{k}\right| \\
& \leq 2 \sum_{|k| \geq \frac{N}{2}}\left|\hat{f}_{k}\right| \leq \frac{2(1+\beta)}{\beta} \cdot\|f\|_{1+\beta} \cdot(1+\beta)^{-\frac{N}{2}}
\end{aligned}
$$


Let us denote by $u(\cdot ; h)$ the solution of the boundary value problem

$$
\left\{\begin{array}{rlr}
\Delta u=0 & \text { in } & \Omega \\
u=h & \text { on } & \partial \Omega
\end{array}\right.
$$

and by $u_{N}(\cdot ; h)$ its MFS approximation. Note that $u_{N}$ depends also on $R$ and the angular parameter $\alpha$. Since both $u(\cdot ; h)$ and $u_{N}(\cdot ; h)$ depend linearly on $h$ we have that

$$
\left\|u_{N}(\cdot ; f)-u(\cdot ; f)\right\|_{\infty} \leq\left\|u\left(\cdot ; f-f_{N}\right)\right\|_{\infty}+\left\|u_{N}\left(\cdot ; f-f_{N}\right)\right\|_{\infty}+\left\|u_{N}\left(\cdot ; f_{N}\right)-u\left(\cdot ; f_{N}\right)\right\|_{\infty} .
$$

Clearly, since $f$ and $f_{N}$ agree at the boundary points, the middle term $u_{N}\left(\cdot ; f-f_{N}\right)$ is identically zero. In what follows we shall show that, each of the remaining two terms on the right hand side of (4.7), decays exponentially fast as $N$ tends to infinity.

\section{A. The term $\left\|u\left(\cdot ; f-f_{N}\right)\right\|_{\infty}$}

Since $u$ is harmonic, it satisfies the maximum principle in $\bar{\Omega}$

$$
\left\|u\left(\cdot ; f-f_{N}\right)\right\|_{\infty}=\sup _{\vartheta \in[0,2 \pi]}\left|\left(f-f_{N}\right)(\vartheta)\right| .
$$

Using (4.6) we obtain that

$$
\left\|u\left(\cdot ; f-f_{N}\right)\right\|_{\infty} \leq \frac{2(1+\beta)}{\beta} \cdot\|f\|_{1+\beta} \cdot(1+\beta)^{-\frac{N}{2}} .
$$

B. The term $\left\|u_{N}\left(\cdot ; f_{N}\right)-u\left(\cdot ; f_{N}\right)\right\|_{\infty}$

Let $f_{N}(\vartheta)=\sum_{k=-\frac{N}{2}+1}^{\frac{N}{2}} \varphi_{k} \mathrm{e}^{i k \vartheta}$. Then

$$
u\left(\cdot ; f_{N}\right)=\sum_{k=-\frac{N}{2}+1}^{\frac{N}{2}} \varphi_{k} u\left(\cdot ; \mathrm{e}^{i k \vartheta}\right) \quad \text { and } \quad u_{N}\left(\cdot ; f_{N}\right)=\sum_{k=-\frac{N}{2}+1}^{\frac{N}{2}} \varphi_{k} u_{N}\left(\cdot ; \mathrm{e}^{i k \vartheta}\right)
$$

Thus

$$
\left\|u_{N}\left(\cdot ; f_{N}\right)-u\left(\cdot ; f_{N}\right)\right\|_{\infty} \leq \sum_{k=-\frac{N}{2}+1}^{\frac{N}{2}}\left|\varphi_{k}\right| \cdot\left\|u_{N}\left(\cdot ; \mathrm{e}^{i k \vartheta}\right)-u\left(\cdot ; \mathrm{e}^{i k \vartheta}\right)\right\|_{\infty} .
$$

The observation which enables us to obtain the desired bound, is the fact that the right hand side of (3.2) reduces to a single term when $h=\mathrm{e}^{i k \vartheta}$, which is easy to treat.

Lemma 4.1. We have the following estimates for $\left\|u_{N}\left(\cdot ; \mathrm{e}^{i k \vartheta}\right)-u\left(\cdot ; \mathrm{e}^{i k \vartheta}\right)\right\|_{\infty}$ :

(i) Case I, $k=0$ : For $R>1$ and every $N \in \mathbb{N}$

$$
\left\|u_{N}(\cdot ; 1)-u(\cdot ; 1)\right\|_{\infty} \leq \frac{8}{N \log R \cdot R^{N}}
$$

(ii) Case II, $0<|k| \leq N / 2$ : For $R>1$ and sufficiently large $N$

$$
\left\|u_{N}\left(\cdot ; \mathrm{e}^{i k \vartheta}\right)-u\left(\cdot ; \mathrm{e}^{i k \vartheta}\right)\right\|_{\infty} \leq 16\left(1-\frac{1}{\sqrt{R}}\right)^{-1} \cdot \frac{|k|}{(N-|k|)} \cdot \frac{1}{R^{N-2|k|}} .
$$


Proof. Case I. $k=0$

In this case $h \equiv 1, \boldsymbol{h}=(1,1, \ldots, 1), u \equiv 1$ and

$$
\left\langle\boldsymbol{h}, \boldsymbol{\xi}_{j}\right\rangle=\left\{\begin{array}{cl}
\sqrt{N} & \text { if } j=1 \\
0 & \text { otherwise. }
\end{array}\right.
$$

Thus from (3.2) we obtain

$$
u_{N}(x, y ; 1)=\sum_{j=1}^{N} \frac{1}{\lambda_{j}}\left\langle\boldsymbol{h}, \boldsymbol{\xi}_{j}\right\rangle \overline{\left\langle\boldsymbol{l}(x, y), \boldsymbol{\xi}_{j}\right\rangle}=\frac{\sqrt{N}}{\lambda_{1}} \overline{\left\langle\boldsymbol{l}(x, y), \boldsymbol{\xi}_{1}\right\rangle}
$$

On the other hand if $(x, y)=(r \cos \phi, r \sin \phi)$ and $r<1$, we have

$$
\left\langle\boldsymbol{l}(r \cos \phi, r \sin \phi), \boldsymbol{\xi}_{1}\right\rangle=\frac{1}{\sqrt{N}} \sum_{j=1}^{N} l_{j}(r \cos \phi, r \sin \phi)
$$

where $\boldsymbol{l}=\left(l_{1}, \ldots, l_{N}\right)($ see $(3.1))$ and if we set $\vartheta_{j, \alpha}=\frac{2 \pi}{N}(j-1+\alpha)$ we obtain

$$
\begin{aligned}
l_{j}(r \cos \phi, r \sin \phi) & =-\frac{1}{4 \pi} \log \left(R^{2}-2 r R \cos \left(\vartheta_{j, \alpha}-\phi\right)+r^{2}\right) \\
& =-\frac{1}{2 \pi} \log R+\frac{1}{2 \pi} \sum_{m=1}^{\infty} \frac{1}{m}\left(\frac{r}{R}\right)^{m} \cos m\left(\vartheta_{j, \alpha}-\phi\right) .
\end{aligned}
$$

Imitating the proof of Theorem 2.6 we obtain

$$
\left\langle\boldsymbol{l}(r \cos \phi, r \sin \phi), \boldsymbol{\xi}_{1}\right\rangle=-\frac{\sqrt{N}}{2 \pi}\left\{\log R+\sum_{m=1}^{\infty} \frac{1}{m N}\left(\frac{r}{R}\right)^{m N} \cos \left(m N\left(\phi-\frac{2 \pi}{N} \alpha\right)\right)\right\}
$$

Similarly, for $0<|k|<N / 2$, we have

$$
\begin{gathered}
\left\langle\boldsymbol{l}(r \cos \phi, r \sin \phi), \boldsymbol{\xi}_{k+1}\right\rangle= \\
\frac{\sqrt{N}}{4 \pi} \sum_{m=0}^{\infty}\left\{\left(\frac{r}{R}\right)^{k+m N} \frac{\mathrm{e}^{-i(k+m N)\left(\phi-\frac{2 \pi}{N} \alpha\right)}}{k+m N}+\left(\frac{r}{R}\right)^{N-k+m N} \frac{\mathrm{e}^{i(N-k+m N)\left(\phi-\frac{2 \pi}{N} \alpha\right)}}{N-k+m N}\right\} .
\end{gathered}
$$


Consequently, using (4.12) and (2.9), we have

$$
\begin{aligned}
u_{N}(x, y ; 1) & =\frac{\sqrt{N}\left\{-\frac{\sqrt{N}}{2 \pi} \log R+\frac{\sqrt{N}}{2 \pi} \sum_{m=1}^{\infty} \frac{1}{m N}\left(\frac{r}{R}\right)^{m N} \cos \left(m N\left(\phi-\frac{2 \pi}{N} \alpha\right)\right)\right\}}{-\frac{1}{4 \pi} \log \left(R^{2 N}-2 R^{N} \cos (2 \pi \alpha)+1\right)} \\
& =\frac{\log R-\sum_{m=1}^{\infty} \frac{1}{m N}\left(\frac{r}{R}\right)^{m N} \cos \left(m N\left(\phi-\frac{2 \pi}{N} \alpha\right)\right)}{\frac{1}{2 N} \log \left(R^{2 N}-2 R^{N} \cos (2 \pi \alpha)+1\right)} \\
& =1+\frac{\left\{\log R-\sum_{m=1}^{\infty} \frac{r^{m N} \cos (\phi-2 m \pi \alpha)}{m N R^{m N}}\right\}-\left\{\log R-\sum_{m=1}^{\infty} \frac{\cos (2 m \pi \alpha)}{m N R^{m N}}\right\}}{\log R-\sum_{m=1}^{\infty} \frac{\cos (2 m \pi \alpha)}{m N R^{m N}}} .
\end{aligned}
$$

Since $u_{N}-u$ is harmonic and $u(\cdot ; 1) \equiv 1$, using the maximum principle we have

$$
\begin{aligned}
\left\|u_{N}(\cdot ; 1)-u(\cdot ; 1)\right\|_{\infty} & =\max _{\vartheta \in[0,2 \pi]}\left|u_{N}(\cos \vartheta, \sin \vartheta ; 1)-1\right| \\
& \leq \max _{\vartheta \in[0,2 \pi]} \frac{\left.\frac{1}{N} \sum_{m=1}^{\infty} \frac{1}{m R^{m N}} \cdot \mid \cos (m N \phi-2 m \pi \alpha)\right)-\cos (2 m \pi \alpha) \mid}{\log R-\sum_{m=1}^{\infty} \frac{1}{m N R^{m N}}} \\
& \leq \frac{2 \frac{1}{N} \sum_{m=1}^{\infty} \frac{1}{R^{m N}}}{\frac{1}{2} \log R}=\frac{4}{N \log R\left(R^{N}-1\right)} \leq \frac{8}{N \log R \cdot R^{N}},
\end{aligned}
$$

for $N$ sufficiently large, i.e. there exists some $N_{1}=N_{1}(R)$, such that the above holds for $N \geq N_{1}$. (Indeed, eventually $1-\frac{1}{R^{N}} \geq \frac{1}{2}$ and $\sum_{m=1}^{\infty} \frac{1}{m N R^{m N}} \leq \frac{1}{2} \log R$.)

Case II. $0<|k|<\frac{N}{2}$

Let us first treat the case $k>0$. Then for $h=\mathrm{e}^{i k \vartheta}$, combining (5.2) and (4.13) we obtain

$$
u_{N}\left(r \cos \phi, r \sin \phi ; \mathrm{e}^{i k \vartheta}\right)=\frac{1}{\lambda_{k+1}}\left\langle\boldsymbol{h}, \boldsymbol{\xi}_{k+1}\right\rangle \overline{\left\langle\boldsymbol{l}(r \cos \phi, r \sin \phi), \boldsymbol{\xi}_{k+1}\right\rangle}=\left(r \mathrm{e}^{i \phi}\right)^{k}\left(1+\frac{S}{T}\right),
$$

where

$$
T=\sum_{m=0}^{\infty}\left\{\left(\frac{1}{R}\right)^{m N} \frac{\mathrm{e}^{-i m N \frac{2 \pi}{N} \alpha}}{k+m N}+\left(\frac{1}{R}\right)^{N-2 k+m N} \frac{\mathrm{e}^{i(N-2 k+m N) \frac{2 \pi}{N} \alpha}}{N-k+m N}\right\}
$$

and

$$
S=\sum_{m=0}^{\infty}\left\{\left(\frac{r}{R}\right)^{m N} \frac{\mathrm{e}^{i m N\left(\phi-\frac{2 \pi}{N} \alpha\right)}}{k+m N}+\left(\frac{r}{R}\right)^{N-2 k+m N} \frac{\mathrm{e}^{-i(N+m N)\left(\phi-\frac{2 \pi}{N} \alpha\right)}}{N-k+m N}\right\}-T
$$


Since $u\left(r \cos \phi, r \sin \phi ; \mathrm{e}^{i k \vartheta}\right)=r^{k} \mathrm{e}^{i k \phi}$ (in general, $u\left(r \cos \phi, r \sin \phi ; \mathrm{e}^{i k \vartheta}\right)=r^{|k|} \mathrm{e}^{i k \phi}$ ), we have

$$
\left|u_{N}-u\right|=\left|u_{N}\left(r \cos \phi, r \sin \phi ; \mathrm{e}^{i k \vartheta}\right)-\left(r \mathrm{e}^{i \phi}\right)^{k}\right|=\left|\frac{S}{T}\right|
$$

and $S / T$, as a function of $(x, y)=(r \cos \phi, r \sin \phi)$, is a harmonic function in $B(0, R)$. Thus it takes its absolute supremum in $B(0,1)$ when $r=1$. Thus for $r=1$ we have

$$
\begin{aligned}
|T| & \geq \frac{1}{k}-\sum_{m=1}^{\infty} \frac{1}{k+m N} \cdot \frac{1}{R^{m N}}-\frac{1}{N-2 k} \cdot \frac{1}{R^{N-2 k}} \sum_{m=0}^{\infty} \frac{1}{N-k+m N} \cdot \frac{1}{R^{m N}} \\
& >\frac{1}{k}\left(1-\frac{1}{R^{N}-1}-\frac{1}{R} \cdot \frac{R^{N}}{R^{N}-1}\right) .
\end{aligned}
$$

It is easy to see that there exists a $N_{2}=N_{2}(R)$, such that, for $N \geq N_{2}$, the right hand side of the above inequality becomes larger than $\frac{1}{2 k}\left(1-\frac{1}{\sqrt{R}}\right)$. Therefore, for $N \geq N_{2}$

$$
|T|>\frac{1}{2 k}\left(1-\frac{1}{\sqrt{R}}\right)
$$

On the other hand, for $r=1$

$$
S=\sum_{m=1}^{\infty} \frac{1}{R^{m N}} \cdot \frac{\mathrm{e}^{-i 2 \pi \alpha m}\left(\mathrm{e}^{-i m N \phi}-1\right)}{k+m N}+\sum_{m=0}^{\infty} \frac{1}{R^{m N+N-2 k}} \cdot \frac{\mathrm{e}^{i 2 \pi \alpha(m+1)}\left(\mathrm{e}^{-i(m+1) N \phi}-\mathrm{e}^{-i \frac{4 k \pi \alpha}{N}}\right)}{N-k+m N} .
$$

Consequently we have

$$
|S| \leq \frac{2}{N+k} \cdot \frac{1}{R^{N}-1}+\frac{2}{N-k} \cdot \frac{1}{R^{N-2 k}} \cdot \frac{1}{1-\frac{1}{R^{N}}} .
$$

Similarly, we can find an $N_{3}=N_{3}(R)$, such that, for $N \geq N_{6}$, the right hand side of the above inequality becomes less than $\frac{8}{N-k} \cdot \frac{1}{R^{N-2 k}}$. Thus for $N \geq N_{3}$ and for $R^{N}>2$

$$
|S|<\frac{8}{N-k} \cdot \frac{1}{R^{N-2 k}}
$$

Combining (4.14), (4.15) and (4.16) we obtain that, for every $N \geq N_{4}=\max \left\{N_{1}, N_{2}, N_{3}\right\}$,

$$
\left\|u_{N}\left(\cdot ; \mathrm{e}^{i k \vartheta}\right)-u\left(\cdot ; \mathrm{e}^{i k \vartheta}\right)\right\|_{\infty} \leq 16\left(1-\frac{1}{\sqrt{R}}\right)^{-1} \cdot \frac{k}{N-k} \cdot \frac{1}{R^{N-2 k}}=M_{2} R^{-N+2 k},
$$

where $M_{2}$ depends only on $R$. The case $k<0,|k|<N / 2$, is treated identically since

$$
u_{N}\left(\cdot ; \mathrm{e}^{-i k \vartheta}\right)=\overline{u_{N}}\left(\cdot ; \mathrm{e}^{i k \vartheta}\right) .
$$

Remark 4.1. A more detailed proof of Lemma 4.1 may be found in [13].

From (4.17) we obtain that

$$
\left\|u_{N}\left(\cdot ; \mathrm{e}^{i k \vartheta}\right)-u\left(\cdot ; \mathrm{e}^{i k \vartheta}\right)\right\|_{\infty} \leq M_{2} R^{-N+2|k|} .
$$


Altogether, for $N \geq N_{4}$

$$
\begin{aligned}
\left\|u_{N}\left(\cdot ; f_{N}\right)-u\left(\cdot ; f_{N}\right)\right\|_{\infty} & \leq \sum_{k=-\frac{N}{2}+1}^{\frac{N}{2}}\left|\varphi_{k}\right| \cdot\left\|u_{N}\left(\cdot ; \mathrm{e}^{i k \vartheta}\right)-u\left(\cdot ; \mathrm{e}^{i k \vartheta}\right)\right\|_{\infty} \\
& \leq \sum_{k=-\frac{N}{2}+1}^{\frac{N}{2}} M_{1}\|f\|_{1+\beta}(1+\beta)^{-|k|} \cdot M_{2} R^{-N+2|k|} \\
& \leq 2 M_{1} M_{2}\|f\|_{1+\beta} \frac{1}{R^{N}} \sum_{n=0}^{\left[\frac{N+1}{2}\right]}\left(\frac{R^{2}}{1+\beta}\right)^{n} \\
& \leq 2 M_{1} M_{2}(N+1)\|f\|_{1+\beta} \frac{1}{R^{N}}\left(1+\frac{R^{N+1}}{(1+\beta)^{\frac{N}{2}}}\right) \\
& \leq M_{3} N\|f\|_{1+\beta}\left(R^{-N}+(1+\beta)^{-\frac{N}{2}}\right),
\end{aligned}
$$

where $M_{3}=4 R M_{1} M_{2}$ and depends on $\alpha, \beta$ and $R$. Combining the estimates of the parts A and $\mathrm{B}$ we obtain

$$
\begin{aligned}
\left\|u_{N}(\cdot ; f)-u(\cdot ; f)\right\|_{\infty} \leq\left\|u\left(\cdot ; f-f_{N}\right)\right\|_{\infty}+\left\|u_{N}\left(\cdot ; f_{N}\right)-u\left(\cdot ; f_{N}\right)\right\|_{\infty} \\
\quad \leq \frac{2(1+\beta)}{\beta} \cdot\|f\|_{1+\beta} \cdot(1+\beta)^{-\frac{N}{2}}+M_{3}\|f\|_{1+\beta} N\left(R^{-N}+(1+\beta)^{-\frac{N}{2}}\right),
\end{aligned}
$$

for $N \geq N_{8}$, which provides the main result of this section:

Theorem 4.2. If the boundary data $f$ satisfy (4.2), for some $\beta>0$ (or equivalently the exact solution u can be extended to a harmonic function in an open domain $D$ containing $\bar{B}(0,1+\beta)$ ), then there exists a positive constant $\tilde{M}=\tilde{M}(\beta, R, \alpha)$, such that

$$
\left\|u_{N}(\cdot ; f)-u(\cdot ; f)\right\|_{\infty} \leq \tilde{M}\|f\|_{1+\beta} N \gamma^{N},
$$

for $N \geq \tilde{N}$, where $\tilde{N}$ depends only on $R$, $\alpha$, and where

$$
\gamma=\max \left\{\frac{1}{R},\left(\frac{1}{1+\beta}\right)^{1 / 2}\right\} .
$$

Remark 4.2. It is noteworthy that (4.19) indicates that if $f$ is a trigonometric polynomial, i.e., $f(\vartheta)=$ $\frac{1}{2} a_{0}+\sum_{n=1}^{L}\left(a_{n} \cos n \vartheta+b_{n} \sin n \vartheta\right)$, then for any fixed $\alpha \in\left[0, \frac{1}{2}\right)$,

$$
\left\|u_{N}-u\right\|_{\infty}=O\left(R^{-(N-2 L)}\right) .
$$

Thus the MFS approximation converges to the exact solution, exponentially fast, as $R$ tends to infinity, while $N$ remains constant.

\section{Stability analysis With Respect to $R$ And $N$}

In [12] it was observed that the numerical approximation deteriorates when $R \gg 1$. Clearly (4.21) does not explain this phenomenon. On the contrary, (4.19) shows that if $f$ is a trigonometric polynomial, then 
$\left\|u_{N}-u\right\|_{\infty}=O\left(R^{-N}\right)$. As we shall see in this section, this is caused by the presence of roundoff errors. In what follows we address the question:

How is the approximate solution $u_{N}=u_{N}(\cdot ; f)$ affected when the boundary data $f$ are perturbed by $\delta f$ ? In particular we shall estimate the error

$$
E=\left\|u_{N}(\cdot ; f+\delta f)-u_{N}(\cdot ; f)\right\|_{\infty} .
$$

More specifically, we shall be looking for the optimal constant $C$ for which

$$
\left\|u_{N}(\cdot ; f+\delta f)-u_{N}(\cdot ; f)\right\|_{\infty} \leq C\|\delta f\|_{\infty}
$$

We shall need the following lemmata:

Lemma 5.1. If $\lambda_{j}(\alpha), j=1, \ldots, N$, are the eigenvalues of the matrix $G^{\alpha}$, then

$$
\left\|u_{N}(\cdot ; h)\right\|_{\infty} \leq \frac{N}{2 \pi} \max \{|\log (R-1)|, \log (R+1)\} \cdot \max _{1 \leq j \leq N} \frac{1}{\left|\lambda_{j}(\alpha)\right|} \cdot \sup _{\vartheta \in[0,2 \pi]}|h(\vartheta)| .
$$

Proof. We have already seen that

$$
u_{N}(\cdot ; h)=\sum_{j=1}^{N} \frac{1}{\lambda_{j}(\alpha)}\left\langle\boldsymbol{h}, \boldsymbol{\xi}_{j}\right\rangle \overline{\left\langle\boldsymbol{l}(\cdot), \boldsymbol{\xi}_{j}\right\rangle}
$$

where $\boldsymbol{h}=\left(h_{1}, \ldots, h_{N}\right), \boldsymbol{l}=\left(l_{1}, \ldots, l_{N}\right), h_{j}=h\left(\frac{2 \pi}{N}(j-1)\right), l_{j}=-\frac{1}{2 \pi} \log \left|P(\cdot)-Q_{j}^{\alpha}\right|, j=1, \ldots, N$. Thus

$$
\begin{aligned}
\left|u_{N}(\cdot ; h)\right| & \leq \max _{1 \leq j \leq N} \frac{1}{\left|\lambda_{j}(\alpha)\right|} \cdot \sum_{j=1}^{N}\left|\left\langle\boldsymbol{h}, \boldsymbol{\xi}_{j}\right\rangle\right| \cdot\left|\left\langle\boldsymbol{l}, \boldsymbol{\xi}_{j}\right\rangle\right| \\
& \leq \max _{1 \leq j \leq N} \frac{1}{\left|\lambda_{j}(\alpha)\right|} \cdot\left(\sum_{j=1}^{N}\left|\left\langle\boldsymbol{h}, \boldsymbol{\xi}_{j}\right\rangle\right|^{2}\right)^{\frac{1}{2}} \cdot\left(\sum_{j=1}^{N}\left|\left\langle\boldsymbol{l}, \boldsymbol{\xi}_{j}\right\rangle\right|^{2}\right)^{\frac{1}{2}}=\max _{1 \leq j \leq N} \frac{1}{\left|\lambda_{j}(\alpha)\right|} \cdot\|\boldsymbol{h}\|_{2} \cdot\|\boldsymbol{l}\|_{2} \\
& \leq \max _{1 \leq j \leq N} \frac{1}{\left|\lambda_{j}(\alpha)\right|} \cdot \sqrt{N}|| \boldsymbol{h}\left\|_{\infty} \cdot \sqrt{N}|| \boldsymbol{l}\right\|_{\infty} \\
& \leq N \max _{1 \leq j \leq N} \frac{1}{\left|\lambda_{j}(\alpha)\right|} \cdot \max \left\{\frac{1}{2 \pi}|\log (R-1)|, \frac{1}{2 \pi} \log (R+1)\right\} \cdot \underset{\vartheta \in[0,2 \pi]}{\max }|h(\vartheta)| .
\end{aligned}
$$

We have used that $\|\boldsymbol{v}\|_{2}=\left(\sum_{j=1}^{N}\left|\left\langle\boldsymbol{v}, \boldsymbol{\xi}_{j}\right\rangle\right|^{2}\right)^{\frac{1}{2}}$, for every $\boldsymbol{v} \in \mathbb{C}^{N}$, since $\left\{\boldsymbol{\xi}_{j}\right\}_{j=1, \ldots, N}$ form an orthonormal basis, and also that $\|\boldsymbol{v}\|_{2} \leq \sqrt{N}\|\boldsymbol{v}\|_{\infty}$.

Lemma 5.2. For $R>1$ and $\alpha \in\left[0, \frac{1}{2}\right)$ fixed, the eigenvalues of the matrix $G^{\alpha}$ satisfy the inequalities

$$
\begin{aligned}
\left|\lambda_{1}(\alpha)\right| & \geq \frac{N}{4 \pi} \log R \\
\left|\lambda_{j}(\alpha)\right| & \geq \frac{N}{8(j-1) \pi} \cdot \frac{1}{R^{j-1}}\left(1-\frac{1}{\sqrt{R}}\right), \quad j=2, \ldots,\left[\frac{N+1}{2}\right], \\
\left|\lambda_{\frac{N}{2}+1}(\alpha)\right| & \geq \frac{|\cos \pi \alpha|}{2 \pi} \cdot \frac{1}{R^{\frac{N}{2}}}, \quad \text { if } N \text { is even, }
\end{aligned}
$$

for $N \geq N_{0}=N_{0}(\alpha, R)$. 
Proof. I. For $j=1$ we have that

$$
\lambda_{1}(\alpha)=-\frac{N}{2 \pi} \log R+\frac{N}{2 \pi} \sum_{m=1}^{\infty} \frac{\cos (2 \pi m \alpha)}{m N R^{m N}}
$$

Thus

$$
\begin{aligned}
\left|\lambda_{1}(\alpha)\right| & \geq \frac{N}{2 \pi} \log R-\frac{N}{2 \pi} \sum_{m=1}^{\infty} \frac{1}{m N R^{m N}}>\frac{N}{2 \pi}\left(\log R-\frac{1}{N} \sum_{m=1}^{\infty} \frac{1}{R^{m N}}\right) \\
& =\frac{N}{2 \pi}\left(\log R-\frac{1}{N} \cdot \frac{1}{R^{N}-1}\right) \geq \frac{N}{2 \pi} \cdot \frac{\log R}{2},
\end{aligned}
$$

when $R^{N} \geq 2$ and $N \geq \frac{2}{\log R}$ or equivalently when $N \geq N_{5}=\left[\frac{2}{\log R}\right]+1=N_{5}(R)$.

II. For $2 \leq j \leq\left[\frac{N+1}{2}\right]$, setting $k=j-1$ we have from Theorem 2.6 that

$$
\lambda_{k+1}(\alpha)=\frac{N}{4 \pi} \sum_{m=0}^{\infty}\left\{\frac{1}{m N+k} \cdot \frac{\mathrm{e}^{-i \frac{2 \pi}{N}(m N+k) \alpha}}{R^{m N+k}}+\frac{1}{m N+N-k} \cdot \frac{\mathrm{e}^{i \frac{2 \pi}{N}(m N+N-k) \alpha}}{R^{m N+N-k}}\right\} .
$$

Thus

$$
\begin{aligned}
\left|\lambda_{k+1}(\alpha)\right| & \geq \frac{N}{4 \pi}\left\{\frac{1}{k R^{k}}-\sum_{m=1}^{\infty} \frac{1}{m N+k} \cdot \frac{1}{R^{m+k}}-\sum_{m=0}^{\infty} \frac{1}{m N+N-k} \cdot \frac{1}{R^{m+N-k}}\right\} \\
& \geq \frac{N}{4 \pi} \cdot \frac{1}{R^{k}}\left\{\frac{1}{k}-\frac{1}{N+k} \cdot \frac{1}{R^{N}} \cdot \frac{1}{1-\frac{1}{R^{N}}}-\frac{1}{N-k} \cdot \frac{1}{R^{N-2 k}} \cdot \frac{1}{1-\frac{1}{R^{N}}}\right\} .
\end{aligned}
$$

Clearly $N-k>k, N-2 k \geq 1$ and for $N$ sufficiently large $\frac{1}{\sqrt{R}}<1-\frac{1}{R^{N}}$, in which case

$$
\frac{1}{k}-\frac{1}{N-k} \cdot \frac{1}{R^{N-2 k}} \cdot \frac{1}{1-\frac{1}{R^{N}}}>\frac{1}{k}\left(1-\frac{1}{\sqrt{R}}\right)
$$

and since $\frac{1}{N+k} \cdot \frac{1}{R^{N}} \cdot \frac{1}{1-\frac{1}{R^{N}}} \rightarrow 0$, for $N \rightarrow+\infty$, there is an $N_{5}=N_{5}(R)$, such that

$$
\left|\lambda_{k+1}(\alpha)\right| \geq \frac{N}{4 \pi} \cdot \frac{1}{R^{k}} \cdot \frac{1}{2 k}\left(1-\frac{1}{\sqrt{R}}\right)
$$

for every $N \geq N_{6}$.

III. Finally for $j=\frac{N}{2}+1$, when $N$ is even and $\alpha \neq \frac{1}{2}$ we have

$$
\lambda_{\frac{N}{2}+1}(\alpha)=\frac{N}{2 \pi} \sum_{m=0}^{\infty} \frac{\cos (2 m+1) \pi \alpha}{\left(m N+\frac{N}{2}\right) R^{m N+\frac{N}{2}}}
$$


Thus

$$
\begin{aligned}
\left|\lambda_{\frac{N}{2}+1}(\alpha)\right| & \geq \frac{N}{2 \pi}\left(\frac{|\cos \pi \alpha|}{\frac{N}{2} R^{\frac{N}{2}}}-\sum_{m=1}^{\infty} \frac{1}{\left(m N+\frac{N}{2}\right) R^{m N+\frac{N}{2}}}\right) \\
& \geq \frac{N}{2 \pi}\left(\frac{|\cos \pi \alpha|}{\frac{N}{2} R^{\frac{N}{2}}}-\frac{1}{\frac{3 N}{2} R^{\frac{3 N}{2}}} \cdot \frac{1}{1-\frac{1}{R^{N}}}\right) .
\end{aligned}
$$

Since $|\cos \pi \alpha|>0$, there exists a $N_{7}=N_{7}(\alpha, R)$, such that for $N \geq N_{7}$

$$
|\cos \pi \alpha| \geq \frac{1}{2} \cdot \frac{1}{3 R^{N}} \cdot \frac{1}{1-\frac{1}{R^{N}}}
$$

in which case for every $N \geq N_{7}$ we have that

$$
\left|\lambda_{\frac{N}{2}+1}(\alpha)\right| \geq \frac{N}{2 \pi} \cdot \frac{1}{2} \cdot \frac{|\cos \pi \alpha|}{\frac{N}{2} R^{\frac{N}{2}}}=\frac{|\cos \pi \alpha|}{2 \pi R^{\frac{N}{2}}},
$$

The proof of the lemma is completed by taking $N_{0}=\max \left\{N_{5}, N_{6}, N_{7}\right\}$.

Given that $\lambda_{k}(\alpha)=\bar{\lambda}_{N-k+1}(\alpha)$, we obtain the following bound for the eigenvalues of $G^{\alpha}$ :

Corollary 5.3. When $\alpha \in\left[0, \frac{1}{2}\right)$, and $N \geq N_{0}=N_{0}(\alpha, R)$ (with $N_{0}$ defined as in the previous lemma) we have that

$$
\max _{1 \leq j \leq N} \frac{1}{\left|\lambda_{j}(\alpha)\right|} \leq \max \left\{\frac{4 \pi}{N \log R}, \frac{2 \pi R^{\frac{N}{2}}}{|\cos \pi \alpha|}, 4 \pi R^{\frac{N}{2}}\left(1-\frac{1}{\sqrt{R}}\right)^{-1}\right\} \leq M_{0} R^{\frac{N}{2}}
$$

for a suitable positive constant $M_{0}=M_{0}(\alpha)$.

Combining (5.1) and (5.3) and using the linear dependence of $u_{N}$ on the boundary data, we obtain for $N \geq N_{0}$ that

$$
\left\|u_{N}(\cdot ; f+\delta f)-u_{N}(\cdot ; f)\right\|_{\infty} \leq M_{0} \cdot \frac{N}{2 \pi} \cdot \max \{\mid \log (R-1), \log (R+1)\} \cdot R^{\frac{N}{2}} \cdot\|\delta f\|_{\infty}
$$

Remark 5.1. We can not do much better that the above estimate (in terms of the order of magnitude of the error) since

$$
\begin{aligned}
\lambda_{\frac{N}{2}+1}(0) & =\frac{N}{2 \pi} \sum_{m=0}^{\infty} \frac{1}{\frac{N}{2}+m N} \cdot \frac{1}{R^{\frac{N}{2}+m N}} \leq \frac{N}{2 \pi} \cdot \frac{1}{\frac{N}{2}} \sum_{m=0}^{\infty} \cdot \frac{1}{R^{\frac{N}{2}+m N}} \\
& =\frac{1}{\pi} \cdot \frac{1}{R^{\frac{N}{2}}} \cdot \frac{1}{1-\frac{1}{R^{N}}},
\end{aligned}
$$

thus, in particular for $\alpha=0$,

$$
\left\|\left(G^{0}\right)^{-1}\right\| \geq \max _{j=1, \ldots, N} \frac{1}{\left|\lambda_{j}(0)\right|} \geq R^{\frac{N}{2}}
$$

for sufficiently large $R$ and $N$. If for instance $\delta f=\varepsilon \mathrm{e}^{i \frac{N}{2} \vartheta}$, then

$$
\left\|u_{N}(\cdot, \delta f)\right\|_{\infty} \geq \varepsilon R^{\frac{N}{2}}
$$


Consequently, the exponential convergence of the MFS is meaningful in practice, provided that $R^{\frac{N}{2}}\|\delta f\|_{\infty} \ll 1$.

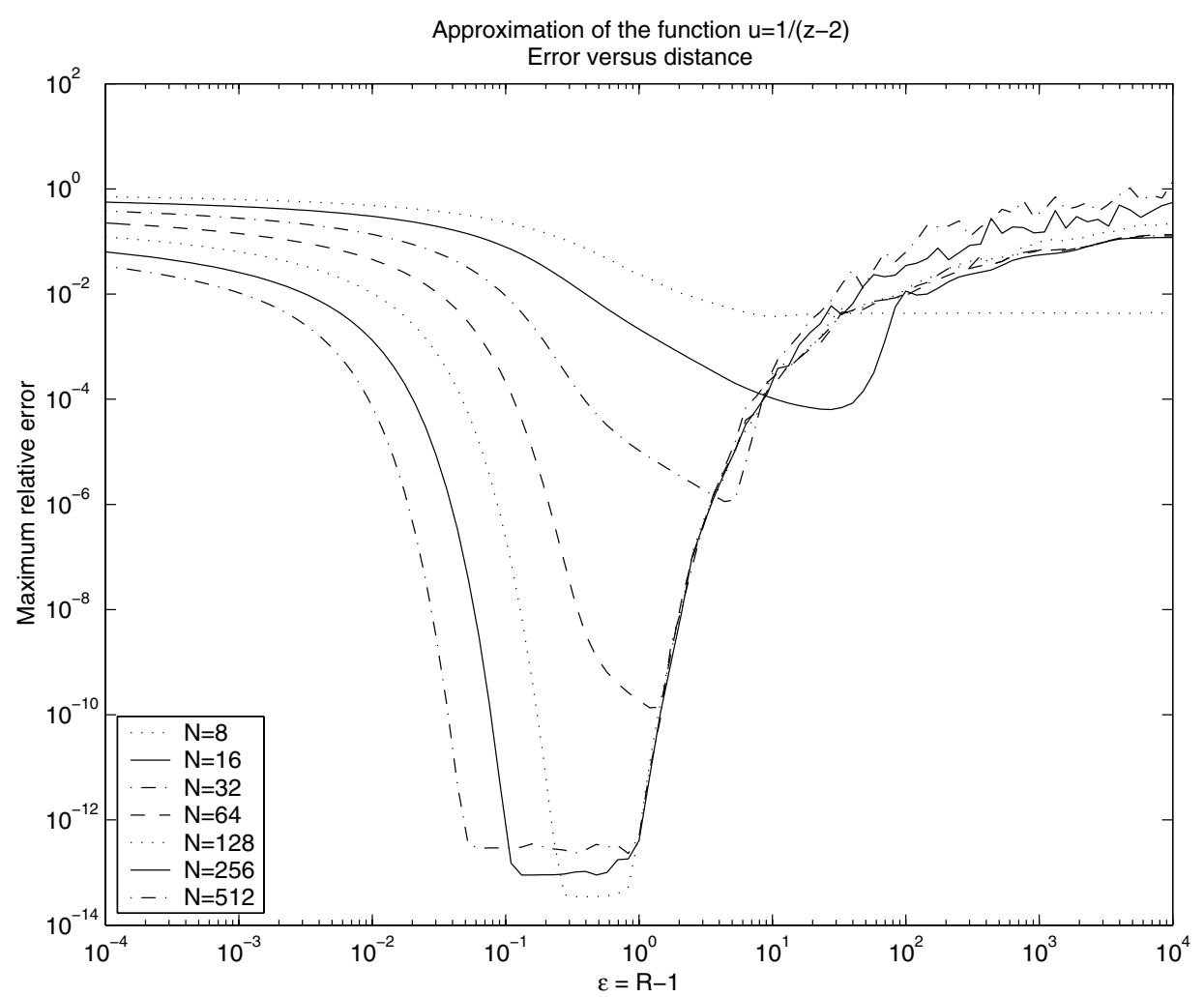

Figure 1. Log of maximum relative error versus $\log \epsilon$ in Example 1.

\section{NumERICAL RESULtS}

The algorithm described in the Section 3 was applied to the problem in $\Omega$ with $\varrho=1$ and exact solution

$$
u=\frac{1}{z-\beta}=\frac{x-\beta}{(x-\beta)^{2}+y^{2}}-i \frac{y}{(x-\beta)^{2}+y^{2}}=u^{R}+i u^{I} .
$$

In particular, we considered the cases with (i) $\beta=2$ (Ex. 1) and (ii) $\beta=1.01$ (Ex. 2). We calculated the maximum relative error

$$
E=\frac{\left\|u^{R}-u_{N}^{R}\right\|_{\infty}+\left\|u^{I}-u_{N}^{I}\right\|_{\infty}}{\|u\|_{\infty}}
$$

where $u_{N}^{R}, u_{N}^{I}$ are the MFS approximations to $u^{R}$ and $u^{I}$, respectively. The maximum relative error was calculated on a uniform grid of $\mathrm{m}$ points on the boundary (since all the functions involved are harmonic and the maximum principle applies) defined by

$$
\left(\cos \theta_{j}, \sin \theta_{j}\right), \theta_{j}=\frac{2 \pi(j-1)}{m}, j=1, \cdots, m .
$$

The parameter $m$ is taken to be equal to 1001 . 


\subsection{Example 1}

In this example the singularity in the solution occurs at the point $(2,0)$ in the (complex) plane and we therefore expect the accuracy to suffer when $R>2$. In Figure 1, we present the behaviour of the maximum relative error for different values of $N$ when $\alpha=0$ for $\epsilon=R-1 \in\left(10^{-4}, 10^{4}\right)$. As expected, we observe that the accuracy improves with $N$ for $R<2$ but eventually deteriorates (for all $N$ ) beyond $R=2$. The behaviour of the solution is similar for values of $\alpha \neq 0$. In Figure 2, we present the maximum relative error as the angular parameter $\alpha$ varies on [0, $\left.\frac{1}{2}\right]$, for different values of $N$ and fixed $\epsilon=10^{-1}, 10^{-2}$ and $10^{-4}$. We observe that the error is optimized for a value of $\alpha \in\left[\frac{1}{6}, \frac{1}{2}\right]$, with the improvement being sharper for the smaller values of $\epsilon$. For $\epsilon=10^{-1}$ and larger, the minimum eventually disappears.
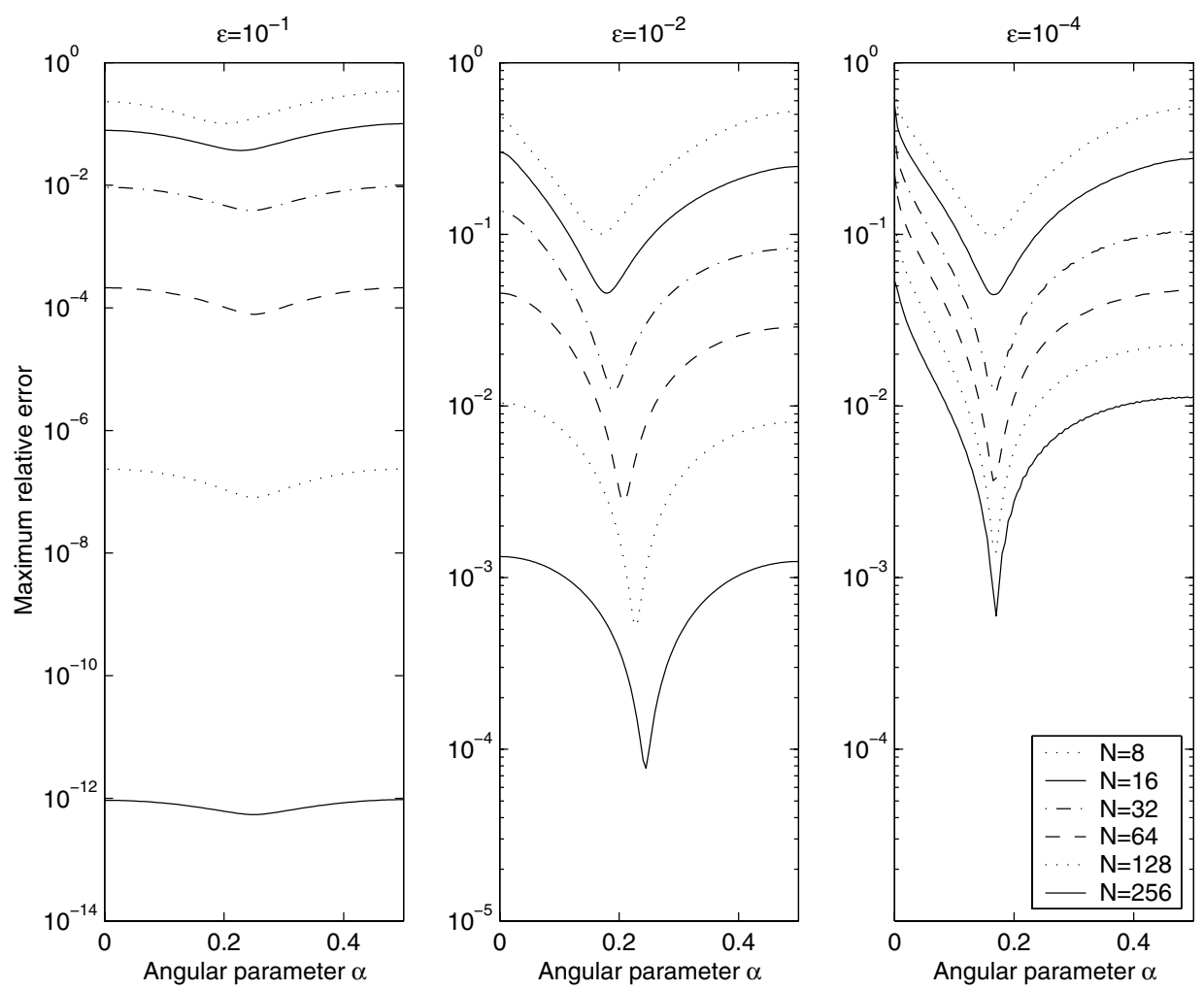

Figure 2. Log of maximum relative error versus $\alpha$ for $\epsilon=10^{-1}, 10^{-2}, 10^{-4}$ in Example 1.

\subsection{Example 2}

In this example the singularity in the solution occurs at the point $(1.01,0)$ in the (complex) plane and we therefore expect the accuracy to suffer when $R>1.01$. As the accuracy of the solution improves with $R$, this leaves very little room for the solution to become accurate and we therefore expect the accuracy of the solution to be poor. In Figure 3, we present the behaviour of the maximum relative error for different values of $N$ when $\alpha=0$ for $\epsilon=R-1 \in\left(10^{-6}, 1\right)$. As expected, we observe that the accuracy of the solution is poorer than in the previous case, with the best results obtained for very small values of $R$. We also observe the eventual deterioration of the solution for $\epsilon>0.01$. In Figure 4, we present the maximum relative error as the angular parameter $\alpha$ varies on $\left[0, \frac{1}{2}\right]$, for different values of $N$ and fixed $\epsilon=10^{-2}, 10^{-3}$ and $10^{-5}$. We observe that the error is minimized only in the cases when $R=10^{-3}$ and $R=10^{-5}$ and the larger values of $N$. In these cases, the point where the error is minimized is close to $\alpha=\frac{1}{6}$. 


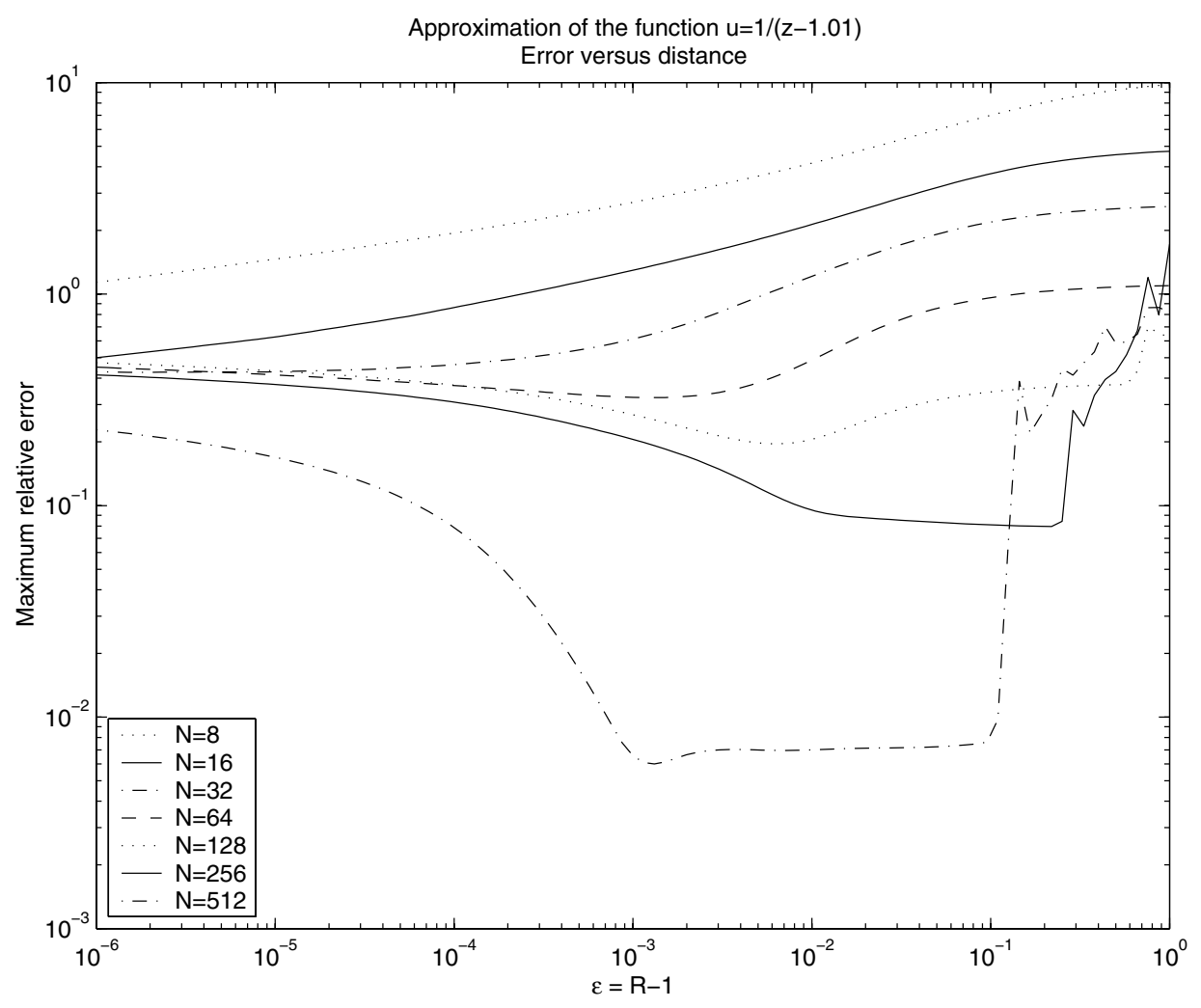

FigurE 3. Log of maximum relative error versus $\log \epsilon$ in Example 2.

\section{Conclusions}

In this work we analyze the MFS when applied to Dirichlet harmonic problems on a disk. Although this is a very specific problem, by exploiting its symmetries we investigate its applicability and present a complete error and stability analysis. These analyses enable us to identify the mechanisms responsible for the rapid convergence of the method for analytic boundary data and the potentially poor stability when the singularities and placed far from the boundary. These features persist for more general domains and different governing elliptic equations.

We examine the properties of the eigenvalues of the matrix arising when the MFS is applied to the above problem. It is shown that these eigenvalues depend on the number of boundary points and singularities $N$, the angular rotation coefficient $\alpha$ and the radius $R$ of the circle on which the singularities are placed. In particular it is shown that the only eigenvalues that can vanish are: (i) the first eigenvalue $\lambda_{1}$, and, (ii) in the case $N$ is even, the eigenvalue $\lambda_{\frac{N}{2}+1}$. Also, expressions for all eigenvalues are given as functions of $N, \alpha$ and $R$. Further, propose an efficient numerical algorithm for the solution of the problem which is designed to overcome the cases when the MFS matrix might be singular. We show that for analytic boundary data, the MFS approximation converges to the exact solution exponentially, with respect to $N$ and we explain why the results obtained with the MFS deteriorate for large $R$ and $N$. 

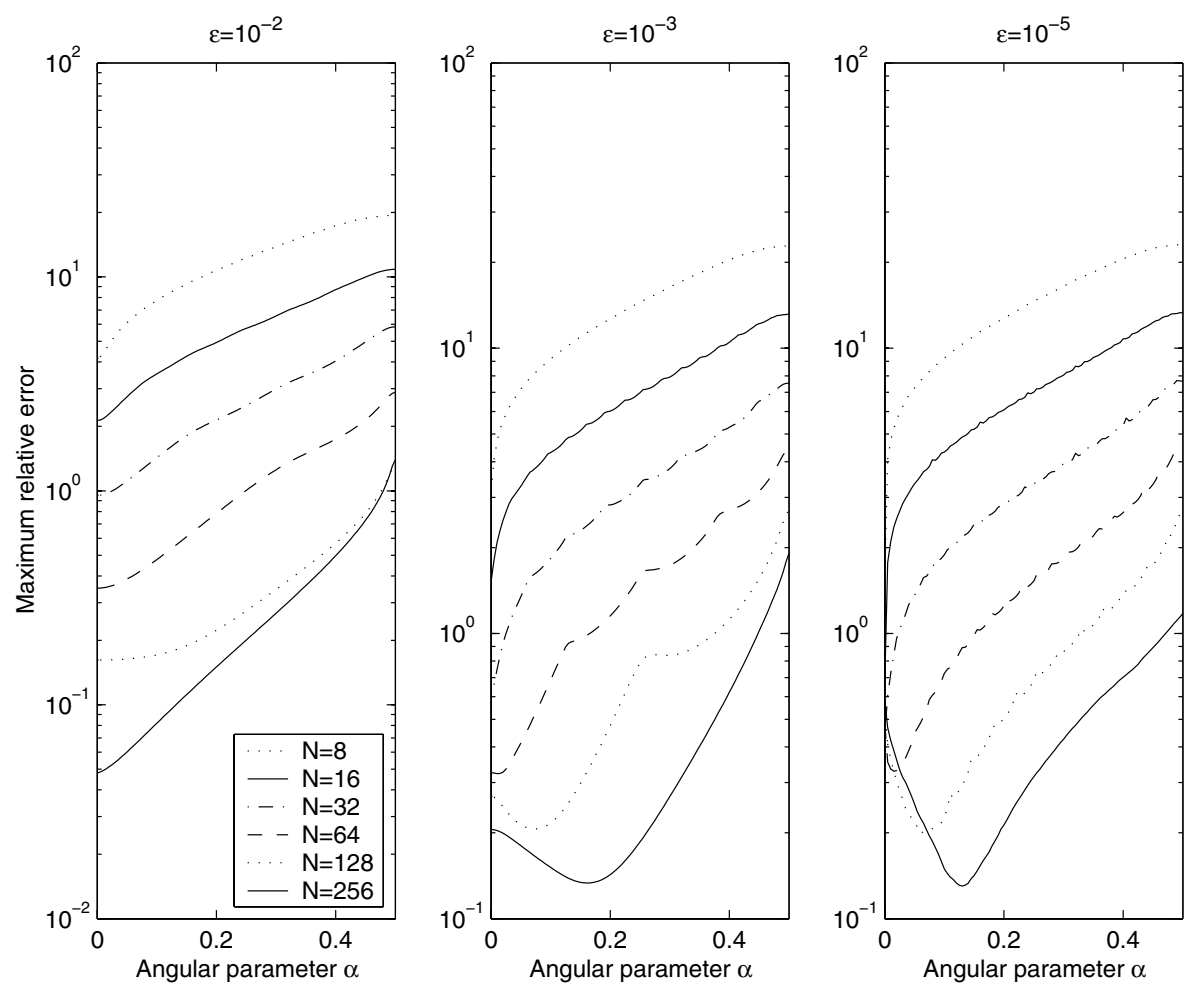

FiguRE 4. Log of maximum relative error versus $\alpha$ for $\epsilon=10^{-2}, 10^{-3}, 10^{-5}$ in Example 2.

\section{REFERENCES}

[1] P.J. Davis, Circulant Matrices, John Wiley \& Sons, New York (1979).

[2] A. Doicu, Y. Eremin and T. Wriedt, Acoustic and Electromagnetic Scattering Analysis Using Discrete Sources. Academic Press, New York (2000).

[3] G. Fairweather and A. Karageorghis, The method of fundamental solutions for elliptic boundary value problems. Adv. Comput. Math. 9 (1998) 69-95.

[4] G. Fairweather, A. Karageorghis and P.A. Martin, The method of fundamental solutions for scattering and radiation problems. Eng. Anal. Bound. Elem. 27 (2003) 759-769.

[5] M.A. Golberg and C.S. Chen, Discrete Projection Methods for Integral Equations. Computational Mechanics Publications, Southampton (1996).

[6] M.A. Golberg and C.S. Chen, The method of fundamental solutions for potential, Helmholtz and diffusion problems, in Boundary Integral Methods and Mathematical Aspects, M.A. Golberg Ed., WIT Press/Computational Mechanics Publications, Boston (1999) 103-176.

[7] I.S. Gradshteyn and I.M. Ryzhik, Table of Integrals, Series, and Products, Academic Press, London (1980).

[8] M. Katsurada, A mathematical study of the charge simulation method II. J. Fac. Sci., Univ. of Tokyo, Sect. 1A, Math. 36 (1989) $135-162$.

[9] M. Katsurada and H. Okamoto, A mathematical study of the charge simulation method I. J. Fac. Sci., Univ. of Tokyo, Sect. 1A, Math. 35 (1988) 507-518.

[10] J.A. Kolodziej, Applications of the Boundary Collocation Method in Applied Mechanics, Wydawnictwo Politechniki Poznanskiej, Poznan (2001) (In Polish).

[11] R. Mathon and R.L. Johnston, The approximate solution of elliptic boundary-value problems by fundamental solutions. SIAM J. Numer. Anal. 14 (1977) 638-650.

[12] Y.S. Smyrlis and A. Karageorghis, Some aspects of the method of fundamental solutions for certain harmonic problems. $J$. Sci. Comput. 16 (2001) 341-371.

[13] Y.S. Smyrlis and A. Karageorghis, Numerical analysis of the MFS for certain harmonic problems. Technical Report TR/04/2003, Dept. of Math. \& Stat., University of Cyprus. 